\title{
Effects of specimen haemolysis on complete blood count results by Abbott Alinity hq System
}

\author{
Müjgan Ercan*1 ${ }^{* 1}$ Emiş Deniz Akbulut ${ }^{2}$, Nihayet Bayraktar ${ }^{3}$, Şerif Ercan ${ }^{4}$ \\ 'Department of Biochemistry, Afyonkarahisar Health Sciences University, Afyonkarahisar, Turkey \\ ${ }^{2}$ Department of Biochemistry, Ankara City Hospital, Ankara, Turkey \\ ${ }^{3}$ Department of Biochemistry, Faculty of Medicine, Harran University, Sanliurfa, Turkey \\ ${ }^{4}$ Department of Biochemistry, Lüleburgaz State Hospital, Kırklareli, Turkey \\ *Corresponding author: mujganercan@hotmail.com
}

\begin{abstract}
Introduction: The current study aimed to assess the interference of in vitro haemolysis on complete blood count (CBC) using Abbott Alinity hq system, and to determine which haemolysis levels affect the reliability of sample results.

Materials and methods: Blood samples obtained from 25 volunteers in K3-EDTA tubes were divided into four aliquots. The first aliquot was not subjected to any intervention. The second, third and fourth aliquots were passed through a fine needle 2, 4 and 6 times, respectively. Complete blood count was performed by multi-angle polarized scatter separation technology and haemolysis index (HI) was assessed from the plasma samples separated by centrifugation. Five groups were formed according to the $\mathrm{HI}$ values. The percentage biases between the results of non-haemolysed and haemolysed groups were compared with the desirable bias limits from The European Federation of Clinical Chemistry and Laboratory Medicine database and reference change values (RCVs).

Results: In groups 1 to 4 , the effects of haemolysis on $\mathrm{CBC}$ parameters were acceptable comparing to the analytical bias except for lymphocytes (7.26\%-7.42\%), MCH (2.59\%), and MCHC (0.47\%-2.81\%). Results of group 5 (gross haemolysis) showed decreases in HCT(- 4.56\%), RBC (- 4.07\%) count and increase in lymphocyte (11.60\%) count higher than the analytical performance specifications. Moreover, variations in MCH (4.65\%) and MCHC (5.24\%) were exceeding the RCVs.

Conclusions: Gross haemolysis (haemoglobin concentration > $10 \mathrm{~g} / \mathrm{L}$ ) is likely to produce unreliable CBC results on non-pathological samples. Further studies including pathological specimens are needed.
\end{abstract}

Keywords: complete blood count; reference change values; haemolysis; interference

\section{Introduction}

Today, laboratory results are known to have considerable effect on clinical decision-making. Sample quality ensured by appropriate sampling techniques is critical in order to obtain accurate results. Errors occurring in the preanalytical phase are accepted as the main source leading to erroneous results (1). In vitro haemolysis is one of the most common preanalytical error affecting many test results. Release of haemoglobin $(\mathrm{Hb})$ and the other intracellular contents secondary to impaired mem- brane integrity of red blood cells (RBC) may cause artifactually increase or decrease results. Inappropriate techniques for collection, transport and processing of samples are the major causes of in vitro haemolysis. Haemolysis may simply be detected by visual inspection of the serum or plasma component of the sample when it contains enough quantity of $\mathrm{Hb}$ to notice. More recently, automated analysers can quantify cell-free $\mathrm{Hb}$ by photometric measurement at different wavelengths following centrifugation (2-9). 
However, recognition of haemolysis before analysis is unlikely and impractical for complete blood count (CBC) as whole blood specimens are used. For this reason concomitant serum or plasma samples may be evaluated for haemolysis or dramatically increased mean corpuscular haemoglobin concentration (MCHC) may be used as warning for the prevention of unnoticed haemolysis $(10,11)$. Determining the effect of haemolysis on CBC parameters is crucial. Accordingly, defining limits to release the results safely or rejecting the sample to avoid related undesirable events may be achieved.

To date, only a few studies have investigated the effect of haemolysis on CBC parameters. The Alinity hq analyser (Abbott, Santa Clara, USA) employs optical scatter and fluorescence technologies as well as cyanide-free absorption haemoglobinometry to perform CBC (12). Multi angle polarized scatter separation (MAPSS) technology used in this system utilizes seven light scatter detectors and a fluorescence detector to collect a unique signal signature on each cell, without using impedance technic (12). To the best of our knowledge, there is no interference study related with this recently released device in the literature.

Therefore, the current study aimed to assess the interference of in vitro haemolysis on complete blood count using Abbott Alinity hq system, and to determine which haemolysis levels affect the reliability of sample results.

\section{Materials and methods}

\section{Subjects}

This cross-sectional study was conducted at Harran University Faculty of Medicine, Biochemistry Laboratory. A total of 25 volunteers were included in the study between January and March 2021. Ethical approval was taken for the study protocol from the Ethical committee of Harran University Faculty of Medicine and written informed consent was obtained from all participants (Approval Number: 14.09.2020/ HRU/20.16.10).

\section{Methods}

Blood samples were drawn into two $3.0 \mathrm{~mL}$ evacuated tubes containing $\mathrm{K} 3$ - ethylenediaminetetraacetic acid (EDTA) (Ref number:IST413) (Isotherm, Istanbul, Turkey) and mixed gently immediately after collection. Samples were portioned in order to obtain four aliquots of $1.5 \mathrm{~mL}$ from each subject subsequently. The first aliquot did not undergo any intervention while the second, third and fourth aliquots were passed through a small calibre needle (22 gauge, $0.70 \times 38 \mathrm{~mm}$ ) 2, 4 and 6 times, respectively in order to produce scalar amounts of haemolysis according to the Dimeski method (13). Routine CBC was performed in all samples on Abbott Alinity hq System (Abbott, Santa Clara, USA) using the same batch of reagents (Abbott, Santa Clara, USA). We conducted an internal quality control process using 3 levels of Alinity h-series Control 29P (Ref. 04U7212, Abbott, Santa Clara, USA) at 20 days. The haematological parameters analysed were as follows: white blood cell count (WBC), neutrophil (neu), lymphocyte (lym), monocyte (mono), eosinophil (eos), red blood cell $(\mathrm{RBC})$, haemoglobin $(\mathrm{Hb})$, haematocrit $(\mathrm{HCT})$, mean corpuscular volume (MCV), mean corpuscular haemoglobin $(\mathrm{MCH})$, mean corpuscular haemoglobin concentration $(\mathrm{MCHC})$, red cell distribution width (RDW), platelet (PLT) and mean platelet volume (MPV).

Afterwards, all aliquots were centrifuged at 1000xg for 10 minutes. Haemolysis index was measured in plasma samples on Siemens Atellica CH chemistry analyser (Siemens Healthineers, Erlangen, Germany). The $\mathrm{HI}$ had 7 semi-quantitative values $(0-6)$ corresponding to $\mathrm{Hb}$ concentrations of $\leq 0.10,0.11$ 1.30, 1.31-2.49, 2.50-4.99, 5.00-7.49, 7.50-9.99 and $\geq 10 \mathrm{~g} / \mathrm{L}$, respectively. All samples were grouped according to $\mathrm{HI}$ values.

\section{Statististical analysis}

The Bland-Altman plot analysis is used to asses the biases between the results of paired groups. Effect of haemolysis interference was evaluated according to the analytical performance specifications (APS) for desirable bias as obtained from the European Federation of Clinical Chemistry and Labora- 
tory Medicine (EFLM) European Biological Variation Study (14). Reference change values (RCVs) were accepted as clinically acceptable limit and derived from within-subject biological variation $\left(\mathrm{CV}_{\mathrm{W}}\right)$ and analytical variation $\left(\mathrm{CV}_{\mathrm{A}}\right)$ with the formula: $\mathrm{RCV}= \pm 1.96 \times\left(\mathrm{CV}_{\mathrm{A}}^{2}+\mathrm{CV}_{\mathrm{W}}{ }^{2}\right)^{1 / 2}$ (15). Total $\mathrm{CV}_{\mathrm{A}}$ was estimated from the results of internal quality control at three concentration levels. Data analyses were performed using MedCalc Statistical Software version 19.1 (MedCalc Software Ltd, Ostend, Belgium).

\section{Results}

At the beginning, the study included a hundred portions with non-pathological results. Later, 16 portions were excluded from the study because plasma volumes obtained after $\mathrm{CBC}$ testing were inadequate for the assessment of HI. Twenty-five portions had no haemolysis and classified as $\mathrm{HI} 0$ (Hb concentration: $<0.10 \mathrm{~g} / \mathrm{L}, \mathrm{N}=25$ ). The samples were presented as 5 groups according to the $\mathrm{HI}$ values; Group 1: HI 1 (Hb concentration: 0.11-1.30 $\mathrm{g} / \mathrm{L}, \mathrm{N}=10$ ), Group 2: $\mathrm{HI} 2$ (Hb concentration: 1.31$2.49 \mathrm{~g} / \mathrm{L}, \mathrm{N}=17$ ), Group 3: HI 3 (Hb concentration: 2.50-4.99 g/L, N = 16), Group 4: HI 4 (Hb concentration: 5.00-7.49 g/L, N = 7), and group 5: HI $6(\mathrm{Hb}$ concentration: $>10.00 \mathrm{~g} / \mathrm{L}$, gross haemolysis, $\mathrm{N}=$ 9). There were no samples with $\mathrm{HI} 5$ ( $\mathrm{Hb}$ concentration 7.50-10.0 g/L). As the degree of haemolysis was different among the samples due to mechanical trauma and to determine the relative percent bias accurately $\mathrm{HI} 0$ groups were paired separately for each group from the non-haemolysed portions $(\mathrm{N}=25)$. Bias percentages and confidence intervals determined for each paired group are presented in Table 1.

In group 1 there was no significant difference in any of the measured parameters.

Results of group 2 showed that at $\mathrm{HI} 2$ all the parameters, with the exception of $\mathrm{MCHC}$, had alterations meeting the APSs. Percent bias of MCHC [0.47 (- 0.4 to 1.33)] was slightly higher than the error limit ( $\pm 0.4 \%$ ) defined according to EFLM database but was still acceptable when evaluated according to RCV (4.12\%).
In group 3, lymphocyte count and $\mathrm{MCHC}$ results had percent bias values: 7.26 (2.84 to 11.68) and 0.73 ( -0.13 to 1.58$)$ above the APS related error limits ( \pm 6.3 and \pm 0.4 , respectively), but they were acceptable in terms of RCVs $(31.00 \%$ and $4.12 \%$, respectively).

Group 4 demonstrated that for the lymphocyte count, $\mathrm{MCH}$ and MCHC parameters, the percent bias 7.42 (1.45 to 9.08), 2.59 (1.37 to 3.80) and 2.81 (1.61 to 4.03 ) were exceeding the analytical limits ( $\pm 6.3 \%, \pm 1.35 \%$ and $\pm 0.4 \%$, respectively) but all values were acceptable according to RCVs.

For the grossly haemolysed group 5, lymphocyte counts had percent bias of 11.60 (5.57 to 17.62) exceeding the analytically desirable limit $( \pm 6.3 \%)$ but were still lower than RCV (31\%). RBC count and HCT were significantly decreased in grossly haemolysed ( $\mathrm{HI}$ 6) samples as compared with nonhaemolysed ones. The percent bias results of RBC count and HCT (- 4.07 (- 5.52 to - 2.62) and - 4.56 (6.13 to -3.00$)$, respectively) were higher than the analytical bias goals $( \pm 1.8 \%$ and $\pm 1.5 \%$, respectively) but under the RCVs (7.46\% and $8.11 \%)$. However, the results of $\mathrm{MCH}$ and $\mathrm{MCHC}$ in this group were affected significantly according to both of the error limits.

\section{Discussion}

We observed that the effect of 0.11 to $7.49 \mathrm{~g} / \mathrm{L} \mathrm{Hb}$ concentration on $\mathrm{CBC}$ parameters were acceptable comparing to the analytical biases with the exceptions of lymphocytes, MCH, and MCHC. In addition, all the parameters were within $\mathrm{RCV}_{\mathrm{S}}$. On the other hand, gross haemolysis (> $10.00 \mathrm{~g} / \mathrm{L} \mathrm{Hb}$ concentration) led to decreases in HCT, RBC count and an increase in lymphocyte count higher than the desirable bias. Moreover, variations in $\mathrm{MCH}$ and MCHC were exceeding the RCVs. Investigation of the impact of haemolysis on CBC is an important requirement. To our knowledge, this is the first study assessing the haemolysis interference in relation to both analytically and clinically significant error limits on the Abbott Alinity hq system.

In the present study, the effect of haemolysis on PLT count has increased in parallel to the degree of 
TABLE 1. The effects of haemolysis on complete blood count

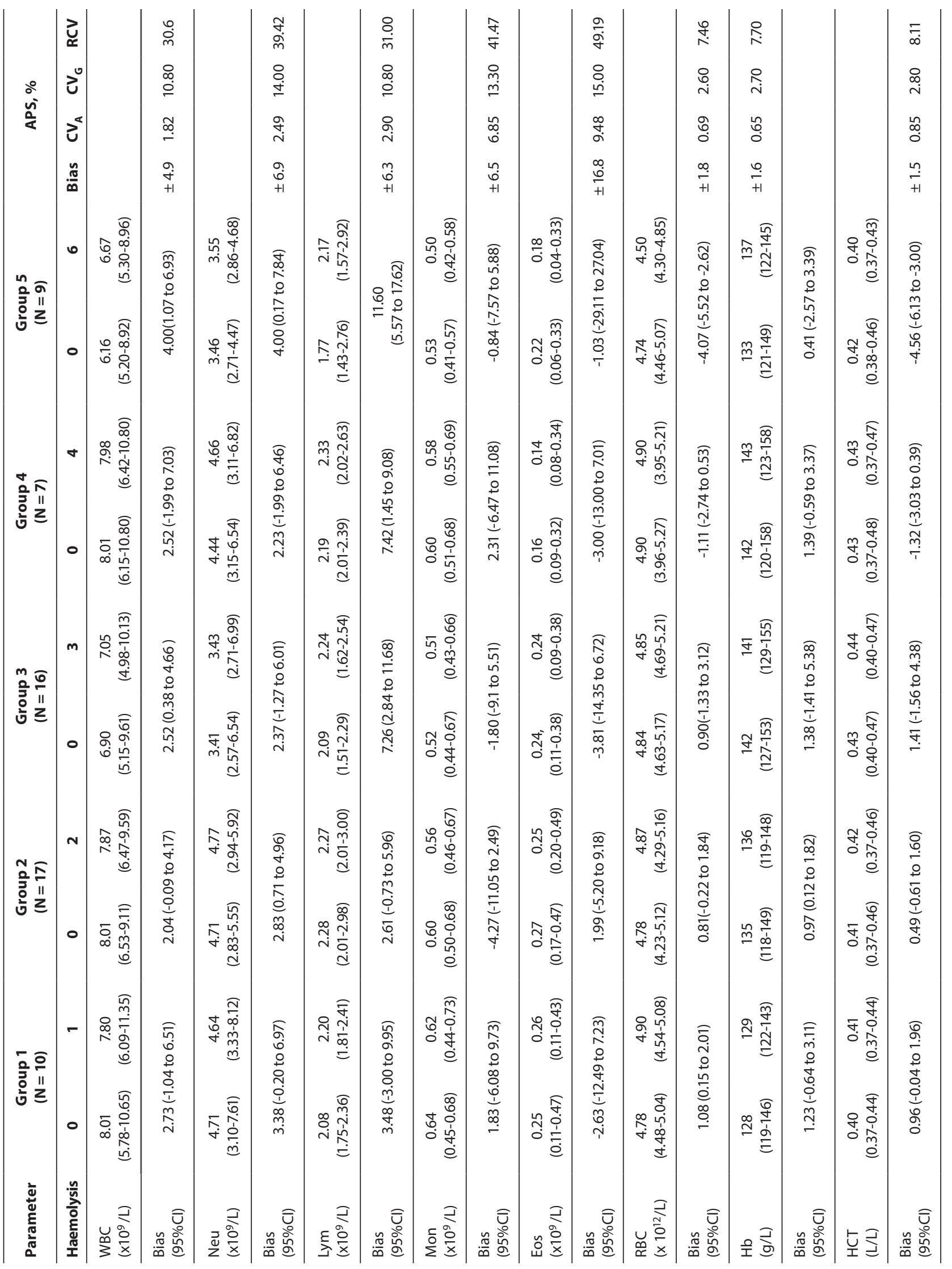




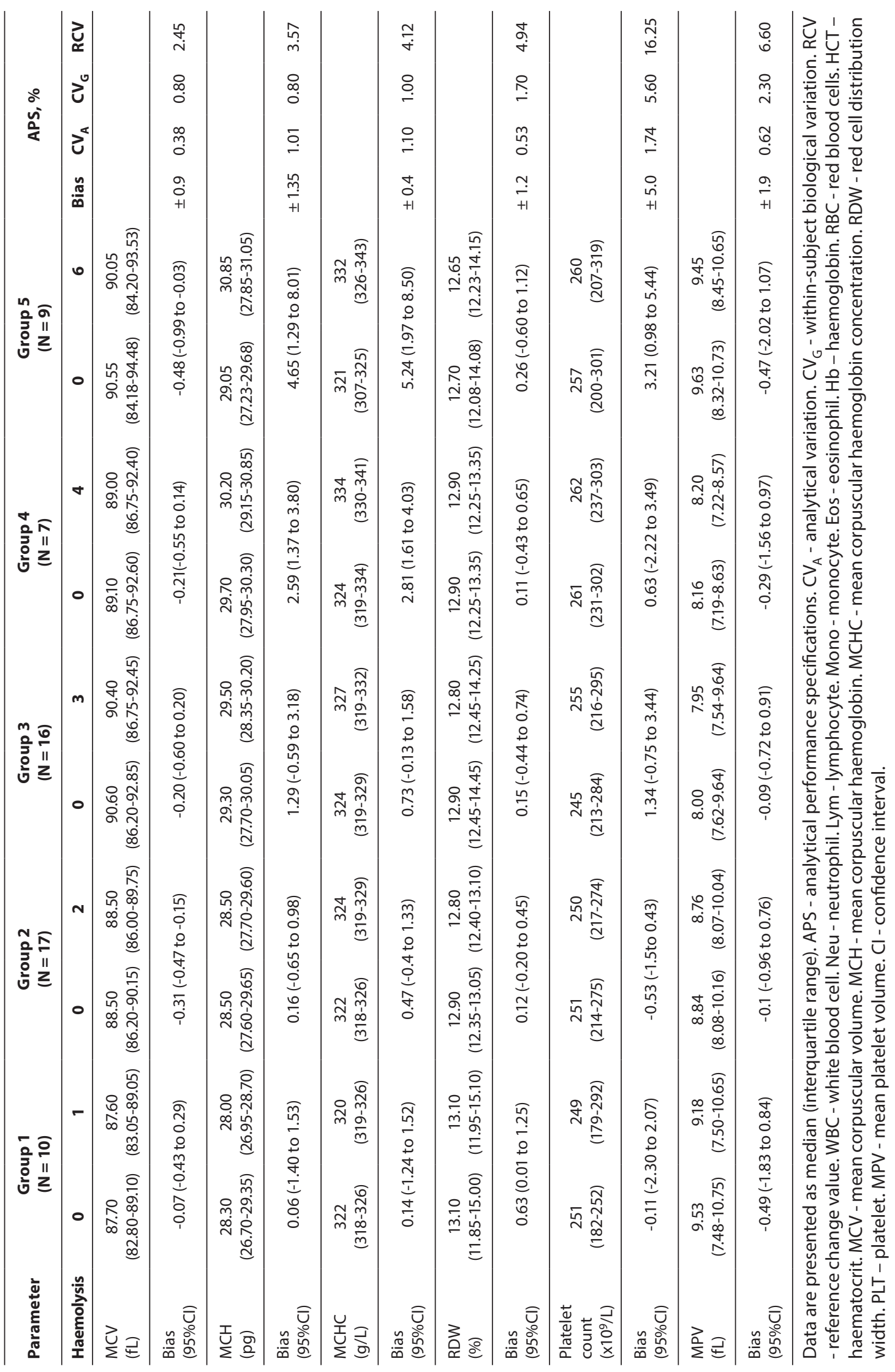


haemolysis. However, it was found to be insignificant comparing to both analytical and clinical error limits. On the other hand, De Jonge et al. indicated that haemolysis caused $7.3 \%$ (- 34.2\% to $48.7 \%)$ and $42.6 \%$ (- $16.8 \%$ to $101.9 \%$ ) bias for PLT count at mild and high degree, respectively (11). In another study it was reported that PLT count was biased $18.1 \%$ (4.2\% to $32 \%)$ and $40.6 \%$ (20.1\% to $61.1 \%$ ) at mild and high degrees of haemolysis (16). One of the possible explanations to this discrepancy between these studies and our study may be the different analytical methodologies used. In the study by De Jonge et al. impedance principle was used (11). Platelets were counted within a specific size range based on the detection of pulses corresponding to the individual cell volume. Since the cell size was the only discriminator and both erythrocytes and PLTs were analysed in the same counting chamber of the analyser, it was suggested that non-platelet particles such as fragmented erythrocytes caused falsely increased PLT counts (17). In the study by Lippi et al., optical light scatter technique was applied for PLT enumeration (16). The instrument had two detectors for the measurement of low and high angle scattered light ensuring two-dimensional PLT analysis. However, the artifactual increase determined in PLT count was attributed to the interference of broken red blood cells and their cytoplasmic fragments $(18,19)$. In the present study PLT count was also determined by an analyser using optical technique. However, this instrument performs measurements at multiple angles with additional detectors for better discrimination.

When RBC counts were evaluated, an analytically significant negative bias was observed for grossly haemolysed samples. Calculated RBC parameters like HCT, MCH and MCHC were also affected due to this decrease in RBC. These results were in compliance with the previous studies $(11,16)$. Most remarkable variations were detected for $\mathrm{MCH}$ and $\mathrm{MCHC}$ in gross haemolysis group exceeding the allowable analytical and clinical bias.

It is reasonable to assume that cell lysis can directly affect RBC count and related indices. However, almost no significant error was determined at $\mathrm{HI}$ values lower than 4 ( $\mathrm{Hb}$ concentration: 0.11-4.99 $\mathrm{g} / \mathrm{L})$ with the exception of MCHC. This was supporting the findings of De Jonge et al. presenting none of RBC parameter outside the acceptable limit at mild haemolysis group obtained by 5 aspirations through a fine needle (11). On the other hand, Lippi et al. found higher bias than the desirable bias for these parameters at mild haemolysis group produced by 5 aspirations corresponding to a $\mathrm{Hb}$ concentration between 4.0-4.5 g/L (16). The possible explanation to this discrepancy might be the difference in the number of times samples passed through the needle to generate mechanical haemolysis. In the present study 2, 4 and 6 times of aspirations caused samples to be haemolysed at 5 different $\mathrm{HI}$ levels; which probably caused by the variabilities among the fragilities of $\mathrm{RBC}$ membranes and their different response to trauma. According to the manufacturer's instruction, approximate concentration ranges for $\mathrm{HI}$ value 1 and 2 were accepted as $0.11-1.30 \mathrm{~g} / \mathrm{L} \mathrm{Hb}$ and 1.31-2.49 $\mathrm{g} / \mathrm{L} \mathrm{Hb}$, respectively. These values were lower than corresponding concentrations expressed in study by Lippi et al. (16). Index value of 3 , which corresponds to a range of $2.50-4.99 \mathrm{~g} / \mathrm{L}$ $\mathrm{Hb}$ concentration, was relatively similar to the previous study's mild haemolysis category. However, this range was also relatively wide and heterogeneous when compared to 4.0-4.5 g/L Hb concentration.

In the $\mathrm{Hb}$ analysis, $\mathrm{RBC}$ sere lysed using a reagent and released $\mathrm{Hb}$ was measured with absorption photometry. Haemoglobin measurement was not affected from haemolysis because the method was not specific for intracellular or free $\mathrm{Hb}$. This finding was concordant with the previous studies $(11,16)$.

The results of this study showed that MCV and RDW (\%) were not interfered significantly with haemolysis comparing to the desirable analytical and clinical bias criteria. This finding was incompatible with the previous studies $(11,16)$. However, these two parameters are known to be interrelated and somewhat dependent to the technology used to measure MCV. In addition, the lack of harmonization between manufacturers in volume distribution curve measurement methodologies, particularly related to exclusion of extreme values 
from the distribution, is suggested to result in differences between analytical systems (20).

Evaluation of WBC counts did not show significant bias in any of the haemolysis groups. Nevertheless, differential analysis revealed that haemolysis led to a positive bias on lymphocytes higher than desirable analytical bias in group 3, 4 and 5. On the other hand, this impact was not significant comparing with RCV.

The present study has some limitations. First, the sample size was relatively low. The study did not include $\mathrm{HI} 5$ (Hb concentration: 7.50-9.99 g/L). In

\section{References}

1. Atay A, Demir L, Cuhadar S, Saglam G, Unal H, Aksun S, et al. Clinical biochemistry laboratory rejection rates due to various types of preanalytical errors. Biochem Med (Zagreb). 2014;24:376-82. https://doi.org/10.11613/BM.2014.040

2. Lippi G, Blanckaert N, Bonini P, Green S, Kitchen S, Palicka V, et al. Haemolysis: an overview of the leading cause of unsuitable specimens in clinical laboratories. Clin Chem Lab Med. 2008;46:764-72. https://doi.org/10.1515/CCLM.2008.170

3. Lippi G. Systematic assessment of the hemolysis index: pros and cons. Adv Clin Chem. 2015;71:157-70. https://doi. org/10.1016/bs.acc.2015.05.002

4. Marques-Garcia F. Methods for hemolysis interference study in laboratory medicine-a critical review. EJIFCC. 2020;31:85-97.

5. Booth J, Pinney J, Davenport A. Changes in red blood cell size and red cell fragmentation during hemodialysis. Int J Artif Organs. 2010;33:900-5. https://doi. org/10.1177/039139881003301209

6. Abed R. Rate of Hematology Specimens Rejection, Association with area of collection and reason of rejection. Int J Biol Med Res. 2013;4:2902-4.

7. Plebani M. The detection and prevention of errors in laboratory medicine. Ann Clin Biochem. 2010;47:101-10. https:// doi.org/10.1258/acb.2009.009222

8. Lippi G, Blanckaert N, Bonini P, Green S, Kitchen S, Palicka $V$, et al. Causes, consequences, detection, and prevention of identification errors in laboratory diagnostics. Clin Chem Lab Med. 2009;47:143-53. https://doi.org/10.1515/ CCLM.2009.045

9. Lippi G, Pavesi F, Benegiamo A, Pipitone S. What do hemolyzed whole-blood specimens look like? Analysis with a CellaVision DM96 automated image analysis system. J Lab Autom. 2015;20:60-3. https://doi. org/10.1177/2211068214559644

10. Lippi G, Plebani M, Di Somma S, Cervellin G. Hemolyzed specimens: a major challenge for emergency departments and clinical laboratories. Crit Rev Clin Lab Sci. 2011;48:143-53. https://doi.org/10.3109/10408363.2011.600228 addition, the different reactions of the erythrocyte membranes of different samples to mechanical trauma might have been generalized with a wider study group. The second limitation of our study is that we could not find the possibility to evaluate haemolysis in pathological specimens.

In conclusion, gross haemolysis (Hb concentration $>10 \mathrm{~g} / \mathrm{L}$ ) is likely to produce unreliable $\mathrm{CBC}$ results in non-pathological samples. Further studies including pathological specimens are needed.

\section{Potential conflict of interest}

None declared.

11. de Jonge $G$, Dos Santos $T L, C r u z B R$, Simionatto $M$, Bittencourt JI, Krum EA, et al. Interference of in vitro hemolysis complete blood count. J Clin Lab Anal. 2018;32:e22396. https://doi.org/10.1002/jcla.22396

12. Woo KS, Jeong IH, An GD, Lim HH, Han JY. Performance evaluation of new Abbott Alinity hq hematology analyzer. Int J Lab Hematol. 2019;41:657-63. https://doi.org/10.1111/ ijlh.13082

13. Dimeski G. Effects of hemolysis on the Roche ammonia method for Hitachi analyzers. Clin Chem. 2004;50:976-7. https://doi.org/10.1373/clinchem.2003.028993

14. Aarsand AK, Fernandez-Calle P, Webster C, Coskun A, Gonzales-Lao E, Diaz-Garzon J, et al. The EFLM Biological Varia tion Database. Available at: https://biologicalvariation.eu/. Accessed August 25th 2021.

15. Fraser CG. Reference change values. Clin Chem Lab Med. 2011;50:807-12. https://doi.org/10.1515/cclm.2011.733

16. Lippi G, Musa R, Avanzini P, Aloe R, Pipitone S, Sandei F. Influence of in vitro hemolysis on hematological testing on Advia 2120. Int J Lab Hematol. 2012;34:179-84. https://doi. org/10.1111/j.1751-553X.2011.01378.x

17. Briggs C, Kunka S, Machin S. The most accurate platelet count on the Sysmex XE-2100. Optical or impedance? Clin Lab Haematol. 2004;26:157-8. https://doi.org/10.1111/j.13652257.2004.00596.x

18. Briggs C, Harrison P, Machin S. Continuing developments with the automated platelet count. Int J Lab Hematol. 2007;29:77-91. https://doi.org/10.1111/j.1751553X.2007.00909.x

19. Mannuß S. Influence of different methods and anticoagulants on platelet parameter measurement. J Lab Med. 2020;44:255-72. https://doi.org/10.1515/ labmed-2020-0037

20. Lippi G, Pavesi F, Bardi M, Pipitone S. Lack of harmonization of red blood cell distribution width (RDW). Evaluation of four hematological analyzers. Clin Biochem. 2014;47:11003. https://doi.org/10.1016/j.clinbiochem.2014.06.003 